\title{
BACK ISOMERIZATION FROM THE EXCITED STATE PHOTOISOMER OF THE LASER DYE 3,3'-DIETHYLOXADICARBOCYANINE IODIDE (DODCI)
}

\author{
Lucla SCAFFARDI, Gabriel'M. BILMES, Daniel SCHINCA and Jorge O. TOCHO \\ Centro de Investigaciones Opticas (CIOn). Comisión de Investigaciones Cientificas de la Provincia de Buenos Aires (CIC). \\ Casilla de Correo 124. 1900 La Plata, Argentina
}

Received 1 May 1987; in final form 14 August 1987

\begin{abstract}
The fuorescence of the first excited state of both the normal (N) and the photoisomer (P) forms of 3,3'-diethyloxadicarbocyanine iodide (DODCl) were analyzed under stcady stale conditions. Argon ion laser and argon ion pumped dye laser were used for excitation. The long wavelength fluorescence, belonging mainly 10 the $\mathrm{P}$ form, shows a similar degree of polarization as the short wavelength part arising from the $N$ form. The huoreseence spectra of DODCl at different fluenees and different wavelengths of taser excitation yielded information about the relative concentrations of the $N$ and $P$ forms. Photocquilibrium between the two species via the excited state of the $P$ form is postulated. The rate constant estimated for the $P^{*} \rightarrow N$ transition is mueh lower than the one expected from a model of a twisted staie through which all the non-radiative transitions of the $P$ lorn take place. The value is in agreement with that derived from the previous oploacoustic measurements exciting with higher flucnees. The results indicate that stimulated emission and excited state absorption are not important under the conditions of the experiment.
\end{abstract}

\section{Introduction}

The study of the photophysics of cyanine dyes has received a great deal of attention in recent years. The main reasons are, on the one hand, their technological use as laser materials or saturable absorbers for mode-locking [1] and, on the other hand, their structural similarity to a variety of natural photobiological systems with polyene structures [2]. 3,3'diethyloxadicarbocyanine iodide (DODCl) is one of the most extensively studied compounds belonging to this group. The knowledge of its behaviour under high-fluence laser excitation is nowadays of great interest because of its fundamental role in the production of femtosecond optical pulses using the colliding pulse technique [3].

The existence of the DODCI photoisomer (P) transient species produced after singlet excitation of the stable form $(N)$, was established by Dempster et al. [4] by laser flash photolysis. The ground state absorption of $\mathrm{P}$ and the isomerization quantum yield were measured and different kinetics paramelers, such as excited state lifetime for both forms and fluorescence quantum yield of $P$ were calculated. Afterwards, the photoisomerization process has been studied by several groups. As a result of these studies a consistent model for the isomerization mechanism is well established $[5,6]$.

The values of the kinetic parameters of the $\mathrm{N}$ form given by Dempster et al. [4] have been confirmed by direct measurements using picosecond pulse excitation [7-9], but only few studies have dealt with the photophysical properties of the $P$ form.

The present paper is concerned with the photophysical processes undergone by excited $P$. We present the results of the laser power dependent fuorescence spectra of DODCI under steady state conditions. Polarization of the fluorescence has been taken into account in the measurements, avoiding instrumental artifacts arising from the emission anisotropy of the fluorophores.

\section{Experimental}

3,3'-diethyloxadicarbocyanine iodide (DODCI) laser dye grade from Kodak was used without further purification. The purity of the dye was checked by thin-layer chromatography on silica plates with dif-

(Norh-Holland Physics Publishing Division) 


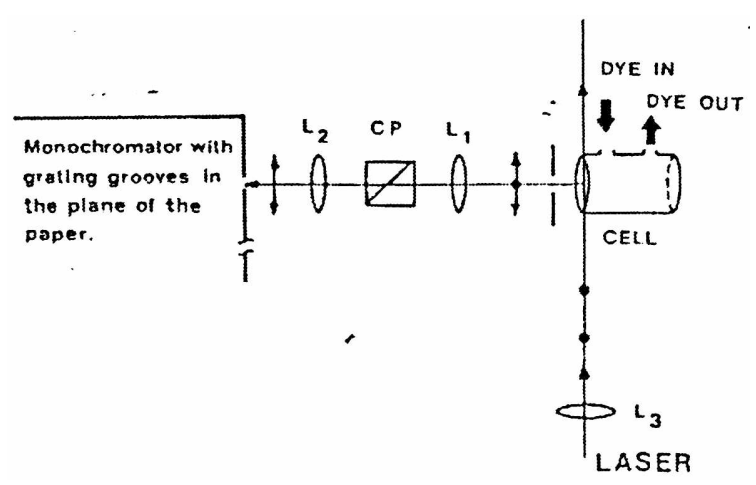

Fig. 1. The experimental apparaius used. Laser is argon ion laser or argon-jon-pumped dye laser. Polarization firections are indicated by dots $(*)$ and double arrows (1). The focal length of $L$, was $67 \mathrm{~mm}$, while $L_{1}$ and $L_{2}$ were arranged 10 focus the fuorescence on the monochromator slit and to illuminate the crystal polarizer $C P$ with low divergence.

ferent solvents. $10^{-6} \mathrm{M}$ solutions in ethanol (Carlo Erba PA) were used immediately after preparation. The solution was circulated at low rate in a cylindrical optical glass cell $(2 \mathrm{~cm}$ diameter flat windows, $5 \mathrm{~cm}$ length). The excitation light from a $\mathrm{cw}$ dye laser tunable between 580 and $620 \mathrm{~nm}$ (Spectra Physics 375 with rhodamine $6 \mathrm{G}$ ) pumped by argon ion laser (SP 165), was focused on the cell by a planoconvex lens $(f=67 \mathrm{~mm})$. The excitation beam passed through the cell as near as possible to the observation window to avoid inner filter effects.

The fluorescence was detected at $90^{\circ}$ with respect to the direction of the excitation beam. The polarization of the pumping beam was perpendicular to the plane defined by the direction of incidence of the excitation beam and the direction of observation of the fluorescence (fig. 1). Some measurements were carried out by exciting the sample with the most intense lines of the argon ion laser ( 488 and $514 \mathrm{~nm}$ ), kceping the same polarization direction.

The spectra were recorded using a $50 \mathrm{~cm}$ focal length Ebert mount scanning spectrometer (Jarrell -Ash 82-025) equipped with a Hamamatsu R-466 multialkali photomultiplier connected to a boxcar integrator (PAR 162/163) triggered internally at 1 $\mathrm{kHz}$. The signals were plotted on an $X-Y$ recorder.

The power was varied by changing the current in the ion pump laser, or by attenuating the dye laser beam on the cell with neutral density filters in the low power region, keeping the laser stabilized well

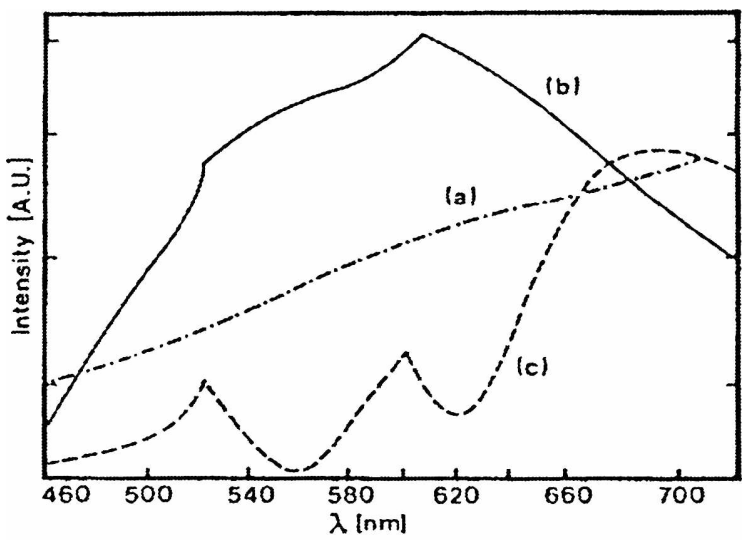

Fig. 2. Calibration of the detcetion systcm. (a) is the calibration lamp specirum, (b) is the response of the system for polarization parallel to the grating grooves and $(c)$ is the response for polarization perpendicular to the grating grooves.

over the threshold. The diameter of the spot on the sample was $50 \mu \mathrm{m}$ as determined by calibrated pinholes. The viewing angle of the fluorescence was properly selected by an iris placed in front of the cell and by the monochromator slit height $(5 \mathrm{~mm})$.

It is well known that the response function of a monochromator-photomultiplier system can difer significanily for polarization parallel or perpendicular to the grating grooves [8]. The system was calibrated by use of a standard tungsten lamp. The spectrum of the lamp (a), and the signals registered by the system for polarization parallel $(b)$ and perpendicular (c) to the grating grooves are shown in fig. 2. From these data, the factors to correct the fluorescence spectra taken under controlled polarization conditions are calculated. Due to the smooth dependence of the detection system (fig. 2) for the parallel to the grating grooves polarization, all the spectra were obtained under these conditions. The emission polarization was selected perpendicular to the excitation polarization by means of a crystal polarizer, $\mathrm{CP}$ in fig. 1.

\section{Results}

The nuorescence spectra of DODCI exhibited a strong dependence with both the fluence and the wavelength of the laser excitation. Typical fluorescence spectra excited at 514 and $609 \mathrm{~nm}$ with dif- 


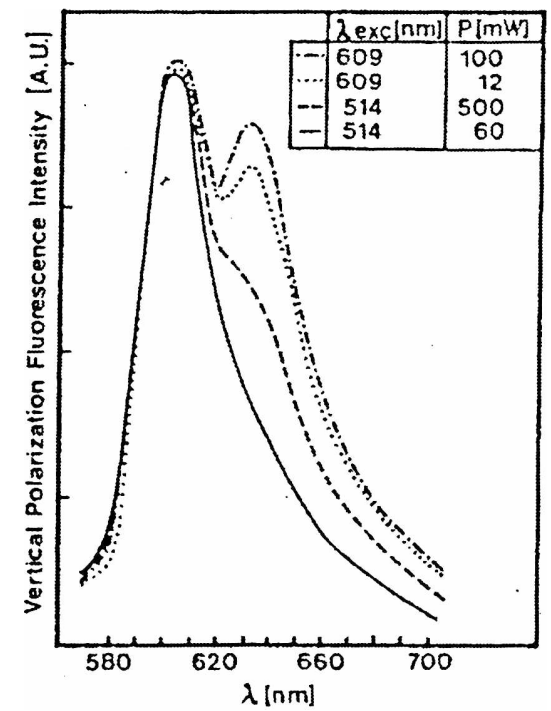

Fig. 3. Typical nuoresecnce spectra of DODCl with different power and wavelengths laser excilation as indicated. The excitation spot diameter was $50 \mu \mathrm{m}$. The spectra are corrected from the iechnical spectra using the factors taken from fig. 2 for polarization parallel to the grating grooves.

- ferent laser powers are shown in fig. 3. The emission intensity is normalized at $595 \mathrm{~nm}$. A laser power of $100 \mathrm{~mW}$ corresponds to a fluence of $1.2 \mathrm{~kW} / \mathrm{cm}^{2}$. As the pump power incrcases, the red fluorescence peak appears and grows in intensity.

The spectra were studied as a function of the rate of ground state absorption by $\mathrm{P}, b=a \sigma^{\mathrm{P}}$, where $\sigma^{\mathrm{P}}$ is the absorption cross section of the P form and $a$ is the photon fluence rate of the excitation. By varying the power and the wavelength of the excitation, the value of $b$ was changed over more than three orders of magnitude. The fluorescence intensities in $\mathrm{W} \mathrm{cm}^{-2}$ $\mathrm{nm}^{-1}, J_{6,0}$ and $I_{(0) 3}$ at 640 and $603 \mathrm{~nm}$ respectively, were selected as representative of the $P^{*}$ and $N^{*}$ emission. These wavelengths correspond to the emission peaks of both species [9]. The ratios between the two intensities, $I_{640} / I_{603}$ for different values of $b$ are given in table 1 .

Due to the emission polarizer only a fraction of the emilled intensily was delected. The fraction emitted with polarization perpendicular to the polarization direction of the excitation is given by $\{(1-p)$, where $p$ is the degree of polarization defined as $p=\left(I_{1}-I_{1}\right) /\left(I_{1}+I_{1}\right)$. The degree of polarization for the DODCl normal form $p_{N}$ was measured at low
Table 1

\begin{tabular}{rllc}
\hline$b\left(\mathrm{~ns}^{-1}\right)$ & $I_{6+0 \mathrm{~nm}} / I_{\mathrm{tD}) \mathrm{nm}}$ & \multicolumn{2}{c}{ Excitation } \\
\cline { 3 - 4 } & & $\lambda(\mathrm{nm})$ & $P(\mathrm{~mW})$ \\
\hline $10^{-6}$ & 0.31 & 514 & 5 \\
$1.2 \times 10^{-3}$ & 0.37 & 514 & 60 \\
$2.4 \times 10^{-9}$ & 0.45 & 514 & 120 \\
$2.5 \times 10^{-3}$ & 0.44 & 600 & 0.6 \\
$5 \times 10^{-3}$ & 0.47 & 514 & 250 \\
& & 514 & 500 \\
$3 \times 10^{-4}$ & 0.57 & 600 & 6 \\
$6 \times 10^{-4}$ & 0.64 & 614 & 6 \\
$8.5 \times 10^{-4}$ & 0.80 & 609 & 12 \\
$2 \times 10^{-4}$ & 0.79 & 614 & 10 \\
$2 \times 10^{-3}$ & 0.81 & 600 & 50 \\
$2.5 \times 10^{-3}$ & 0.73 & 609 & 36 \\
$3 \times 10^{-3}$ & 0.83 & 614 & 30 \\
$7 \times 10^{-3}$ & 0.86 & 609 & 100 \\
\hline
\end{tabular}

power excitation using a spectrofuorimeter (SLM). In ethanol at room temperature, $p_{\mathbb{N}}=0.17$ was obtained. The degree of polarization of the fluorescence emilted at $700 \mathrm{~nm}$ with higher fluence excitation was analyzed with the equipment in fig. 1. Different power and wavelengths in the laser excitation were used and $b$ varied befween $10^{-6}$ and $5 \times 10^{-3} \mathrm{~ns}^{-1}$. The value of $p$ obtained was $0.15 \pm 0.04$, independent of $b$. For the higher values of $b$, most of the intensity comes from the $P$ form at this emission wayelength. The necessity of evaluating the degree of polarization and the use of a polarizer in the detection arise from erratic preliminary results obtained when this factor was not considered.

\section{Discussion}

II is well established that the photoisomerization of DODCI proceeds via the first excited singlet state [10]. The radiationless deactivation of $N^{*}$ occurs through a shortlived twisted state which branches between the ground stable $N$, and that of the less stable $P$. Due to the strong overlap of the absorption of both species, at high fluences the first excited singlet state of $P$ is also populated. $P^{*}$ is responsible of the red fuorescence peak in fig. 3. The mechanism for the non-radiative deactivation of $P^{*}$ is not completely clear. Rulliere suggested a similar mechanism than that of $N^{*}$, that is, a branching between the 


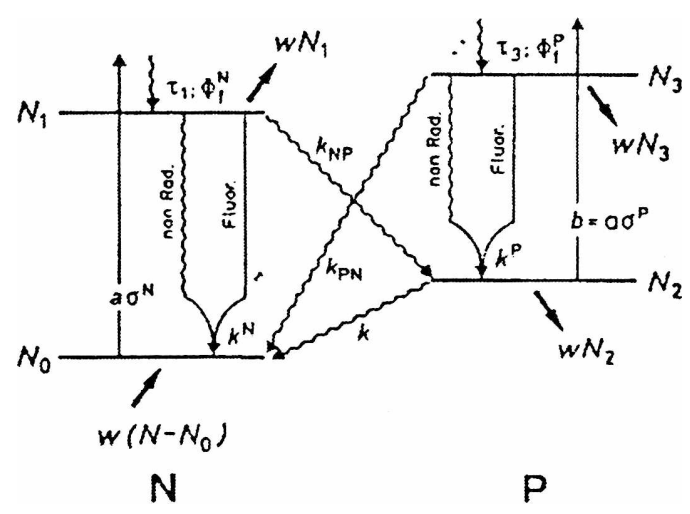

Fig. 4. Four-level scheme used and rate constants for the difterent processes involved. Non-radiative transitions are indicated by wavy lines. $w$ is the rate constant for the replenishment by the circulation.

direct internal conversion to $P$ and the back isomerization to $N[5]$.

The scheme in fig. 4 was used to model the results in table 1. This scheme is equivalent to Rullière's model in the limit of a very short lifetime for the twisted form [6]. The rate constants of the ground state absorption of $N$ and $P$ are represented by $a \sigma^{N}$ and $b=a \sigma^{2}$, respectively. The rate constant for the radiative plus non-radiative transitions between $N^{*}$ and $N$ is given by $k^{N}$. For the $P$ form, $k^{p}$ is the rate constant for the radiative plus the non-radiative transitions between $P^{*}$ and $P$. The rate constants for the interconnections between the $N$ and $P$ forms are given by $k_{\mathrm{NP}}, k_{\mathrm{PN}}$ and $k$ as explained in fig. 4 . The lifetimes, and the fluorescence quantum yields for the $N$ and $P$ forms are indicated by $\tau_{1}, \hat{\phi}_{f}^{N}$, and $\tau_{3}$, $\phi_{\mathrm{f}}^{\mathrm{P}}$, respectively. The change of state population by circulation of the solution is governed by the rate constant $w$. No triplet state was considered due to the small intersystem crossing yield, $\phi_{\mathrm{ISC}}<0.005$ [4] and the efficient quenching by $\mathrm{O}_{2}$ in the air-saturated solutions used.

Under these assumptions, the steady state population of the four levels in fig. 4 can be described by the sel of rate equations:

$-a \sigma^{N} N_{0}+k N_{2}+k_{p N} N_{3}+w\left(N-N_{0}\right)+k^{N} N_{1}=0$,

$a \sigma^{N} N_{0}-N_{1} / \tau_{1}-w N_{1}=0$ $-b N_{2}-k N_{2}+k_{N P} N_{1}+k^{p} N_{3}-w N_{2}=0$,

$b N_{2}-N_{3} / \tau_{3}-w N_{3}=0$,

where $N$ is the number of molecules in state $i$ as indicated in fig. 4 and the total number of molecules is given by

$N=N_{0}+N_{1}+N_{2}+N_{3}$.

Solving (1) $-(5)$ the ratio of the populations of excited $P$ and $N$ is

$\frac{N_{3}}{N_{1}}=\frac{k_{N r} b}{\left.(\tau)^{-1}+w\right)(k+w)+b\left(k_{\mathrm{PN}}+w\right)}$.

Eq. (6) can be used to simulate the results of table 1. The fuorescence intensity emitted at wavelength $\lambda$, in units of $\mathrm{W} \mathrm{cm}^{-2} \mathrm{~nm}^{-1}$ by the two species is

$I_{\lambda}=\left[\left(N_{1} / \tau_{1}\right) E_{N}(\lambda)+\left(N_{3} / \tau_{3}\right) E_{F}(\lambda)\right] h d \lambda$,

where $E_{N}$ and $E_{\mathrm{p}}$ represents the band shape function in photons/nm, of the nuorescence emitted by $N$ and $P$ respectively, normalized to $\int_{0}^{\infty} E_{N, \Gamma}(\lambda) d \lambda=$ $\phi_{1}^{\mathrm{N}}, \phi_{r}^{\mathrm{P}}$. The ratio between the emitted intensities at $\lambda_{1}=640 \mathrm{~nm}$ and $\lambda_{2}=603 \mathrm{~nm}$ can be written as

$\frac{I_{640}}{I_{603}}=\left(\frac{E_{\mathrm{N}}(640)}{E_{\mathrm{N}}(603)}+\frac{N_{3} \phi_{\mathrm{P}}^{\mathrm{P}} \tau_{1}}{N_{1} \phi_{\mathrm{f}}^{\mathrm{N}} \tau_{3}}\right) \frac{603}{640}$,

if $E_{P}(603)=0$ and $E_{\Gamma}(640) / E_{N}(603)=\phi_{\mathrm{r}}^{F} / \phi_{\mathrm{r}}^{N}$ are assumed due to the similarity of the shape of the fluorescence bands. In order to compare the results of eq. (8) with the measurements of table I other fact must be considered: due to the emission polarizer (see fig. 1) the intensities quoted in table 1 are only a fraction of the total intensity emitted. The fraction is governed by the degree of polarization $p$. From the values of $p=0.17$ for low power excitation and $0.15 \pm 0.04$ for high power excitation, it can be assumed that, at room temperature in ethanol, the degree of polarization $p$ for the two isomeric species of DODCI is the same. Under this assumplion and introducing $(6)$ in $(8)$, the ratio of the measured intensities at 640 and $603 \mathrm{~nm}$ is given by

$$
\begin{aligned}
\frac{I_{640}}{I_{603}} & =\left(\frac{E_{N}(640)}{E_{N}(603)}\right. \\
+ & \left.\frac{k_{N P} b\left(\phi_{r}^{\mathrm{P}} \tau_{1} / \phi_{r}^{N} \tau_{3}\right)}{\left(\tau_{3}^{-1}+w\right)(k+w)+b\left(k_{\mathrm{PN}}+w\right)}\right) \frac{603}{640} .
\end{aligned}
$$




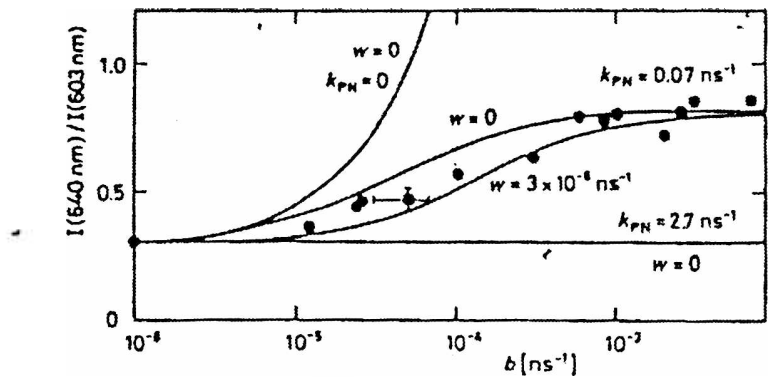

Fig. 5. Filting of the measurements of table 1 by the expression of eq. (9). The best fitting is oblained for $k_{\mathrm{rey}}=0.07 \mathrm{~ns}^{-1}$ and $w \leqslant 3 \times 10^{-6} \mathrm{~ns}^{-1}$. The other parameters in eq. (9) are taken from the literature; the values are given in the text. No connection $\left(k_{\mathrm{rN}}=0\right)$ and strong connection $\left(k_{\mathrm{rN}}=2.7 n 5^{-1}\right)$ between $P^{*}$ and $N$ are in disagreement with the experiment.

- The values of $I_{640} / I_{603}$ calculated with eq. (9) are given in fig. 5 as a function of $b$ for different values of the rate constant for the back isomerization $k_{P N}$ and the circulation parameter $w$. The other parameters are taken from the litetature 14]: $E_{N}(640$ $\mathrm{nm}) / E_{\mathrm{N}}(603 \mathrm{~nm})=0.32, k_{\mathrm{NP}}=0.07 \mathrm{~ns}^{-1}, \tau_{3}=0.3$ $\mathrm{ns}$ and $k=7.7 \times 10^{-7} \mathrm{~ns}^{-1}$. The values obtained under different excitation conditions are best fitted by $k_{\mathrm{PN}}=0.07 \mathrm{~ns}^{-1}$ and $w \leqslant 3 \times 10^{-6} \mathrm{~ns}^{-1}$, in agreement with the values used by Bilmes et al. [11] to explain the results from optoacoustic measurements. The value for $w$ assuming homogeneous flow ( $w=$ circulation speed/excised volume) is $2 \times 10^{-5} \mathrm{~ns}^{-1}$ corresponding to $1 \mathrm{~cm}^{3} / \mathrm{s}$ circulation speed and $4 \times 10^{-3} \mathrm{~cm}^{3}$ excited volume. It is difficult to estimate the exact rate of molecular replenishment due to the turbulent flux near the cell wall; a considerable lower value for $w$ should be expected.

The value $k_{\mathrm{PN}}=0$ in fig. 5 corresponds to no conasion from excited $P$ to the ground state of $N$. A large population of $P$ results and the red fluorescence at $640 \mathrm{~nm}$ is overestimated for most of the $b$ values. The value for $k_{\mathrm{PN}}=2.7 \mathrm{~ns}^{-1}$ was calculated considering that the radiationless deactivation of $\mathrm{N}^{*}$ proceed mainly via a twisted state $[5,6]$, from which a fraction $\beta$ of molecules undergoes isomerization to the $P$ ground state and $1-\beta$ goes back to the $N$ ground statc. The branching ratio $\beta=0.12$ can be estimated from Dempster's measurements [4]. If a similar scheme is assumed for the non-radiative deexcitation of $\mathrm{P}^{*}, k_{\mathrm{PN}}$ can be calculated from
$k_{\mathrm{F}^{\prime} \mathrm{N}}=(1-\beta) k_{\mathrm{NR}}^{\mathrm{P}}=(1-\beta)\left(1-\phi_{\mathrm{f}}^{\mathrm{P}}\right) \tau_{3}^{-1}$,

For $\phi_{\mathrm{f}}^{\mathrm{P}}=0.07$ [4], eq. (10) yields $k_{\mathrm{PN}}=2.7 \mathrm{~ns}^{-1}$. Such strong conversion would result in a constant value for the emission intensity ratio, showing that no relevant population of $P$ ground state is built up. In this case, the fluorescence would come mainly from the $\mathrm{N}$ form, in contrast to the results in figs. 3 and 5.

\section{Conclusions}

The excitation fluence and wavelength dependence of the fluorescence of DODCI, allowed the determination of the back isomerization (reformation) rate constant for the process starting in the first excited singlet state of the less stable $P$ form. $k_{F N}=0.07 \mathrm{~ns}^{-1}$ was calculated in agreement with recently performed laser-induced optoacoustic measurements [11]. This value is much smaller than that calculated using the model of radiationiess deactivation through a twisted state. In contrast to the processes undergone by $N$, the intramolecular twisting is not the dominant non-radiative process for exciled $P$. Direct internal conversion becomes its main nonradiative process.

\section{Acknowledgement}

This work was supported by the Consejo Nacional de Investigaciones Cientificas y Técnicas (CONICET) Grant PID: $3901601 / 85$ and by CIC. We thank Professor S.E. Braslaysky of the Max-Planck-Institut fur Strahlenchemie, Mülheim, FRG, who continuously encourages our work. We are indebled to Professor M. Garavaglia of $\mathrm{ClOp}$ for his support and to Dr. H.F. Ranca-Sandoval for the help in the preparation of the manuscript and fruitful discussions. The degree of polarization of DODCI normal form was determined with Dr. A. Palandini at the Instituto de Investigaciones en Ingenieria Genética, Bue nos Aires. LS and JOT are members of CONICET and $G M B$ and DS are supported by CONICET. 


\section{References}

III W. Sibbett, J.R. Tayior and D. Welford, IEEE J. Quantum Electron. QE-17 (1981) 500, and references therein.

[2] O. Isler, ed., Carolenoids (Birkhäuser, Basel, 1971).

[3] J.A. Valdamis and R.L. Fork, IEEE J. Quantum Electron. QE-22 (1986) 112.

[4] D.N. Dempster, T. Morrow, R. Rankin and G.F. Thompson, J. Chem. Soc. Faraday Trans. II 68 (1972) 1479.

[5] C. Rullière, Chem. Phys. Lelters 43 (1976) 303.
[6] S.P. Velsko and G.R. Fleming, Chem. Phys. 65 (1982) 59. [7] D. Magde and M.W. Windsor, Chem. Phys. Leliers 27 (1974) 31 .

[8] J.M. Miller, ed., Standards in fluorescence spectrometry (Chepman and Hail, London, 1981) ch. 7.

[9] F. Heisel, J.A. Mieht and B. Sipp, J. Luminescence 24 (1981) 651 .

(10) S.K. Rentsch, Chem. Phys. 69 (1982) 81.

[II] G.M. Bilmes, J.O. Toho and S.E. Braslavsky, Chem. Phys. Letiers 134 (1987) 335. 